\title{
SURVEY ON EVOLUTIONARY COMPUTATION TECH TECHNIQUES AND ITS APPLICATION IN DIFFERENT FIELDS
}

\author{
Rajkumari Bidyalakshmi Devi, Esha Barlaskar, Oinam Binarani Devi, \\ Smriti Priya Medhi and Reingayung Ronra Shimray
}

Don Bosco College of Engineering And Technology,Assam Don Bosco University, Guwahati, Assam, India

\begin{abstract}
In computer science, 'evolutionary computation' is an algorithmic tool based on evolution. It implements random variation, reproduction and selection by altering and moving data within a computer. It helps in building, applying and studying algorithms based on the Darwinian principles of natural selection. In this paper, studies about different evolutionary computation techniques used in some applications specifically image processing, cloud computing and grid computing is carried out briefly. This work is an effort to help researchers from different fields to have knowledge on the techniques of evolutionary computation applicable in the above mentioned areas.
\end{abstract}

\section{KEYWORDS}

Cloud Computing, Evolutionary Computation, Grid Computing, Image Processing and Swarm Intelligence.

\section{INTRODUCTION}

Evolutionary computation (EC) is a subfield of artificial intelligence. It is fairly a new vibrant area of investigation which uses ideas of biological evolution to solve computational problems. A computational problem often requires system to be adaptive. Evolution is an optimization process that aims to improve the ability of an organism to survive in dynamically changing environments [1]. EC refers to computer-based problem solving system that uses computational models of evolutionary processes such as Natural Selection, Survival of the fittest, Reproduction as the fundamental component of such computational system. The theory of natural selection purposes that the plants and animals that exist today are the result of millions of years of adaptation to the demands of environment. Fitness of an organism is measured by success of the organism in adapting to its environment. Survival of fitness: individual that are more fit have more chances to survive longer hence more chances to reproduce and propagate there genotype to future generation [2].

The algorithms based on evolutionary principles are the techniques behind evolutionary computation. These algorithms are applicable for searching optimal solution to a problem. There may be a number of possible solutions to a problem in a search algorithm and finding the best/optimal out of these many solutions in a certain limited time can be challenging.

If the numbers of possible solutions are less then lesser amount of time will be consumed in the search for optimal solution. So the drawback is it becomes exhaustible and impractical if the search space is very large. Researchers in many fields face computational problems in which great number of solutions is possible but finding an optimal solution is difficult. A variety of 
International Journal on Information Theory (IJIT), Vol.3, No.3, July 2014

search approach techniques have been developed for exploring such problem spaces. One promising approach of using evolutionary computation technique has been the use of algorithms based upon the principles of natural evolution involving meta-heuristic algorithms. Broadly speaking, the fields include:

\subsection{EVOLUTIONARY ALGORITHM:}

- Genetics expressing programming

- Genetic algorithm

- Genetics programming

- Evolutionary programming

- Evolutionary strategy

- Differential evolution

- Differential search algorithm

\subsection{SWARM INTELLIGENCE}

- Ant colony optimization

- Particle swarm

- Bess algorithm

- Cuckoo search

In this work we are emphasizing on the applications of Evolutionary computation techniques in the fields of Image Processing, Cloud Computing and Grid Computing which would be further described briefly in the following section 2. In section 3 Evolutionary algorithm and its application on Genetic algorithm is discussed based on Image Processing, Cloud Computing and Grid Computing. Further discussion on section 4 describes Swarm Intelligence along with some application on Image Processing and Cloud Computing.

\section{Image Processing, Cloud Computing ANd Grid Computing}

The general idea of image processing is the conversion of an image into its digital form where certain operations can be performed upon it either to obtain an enhanced image or to extract some useful information from it. Evolutionary computation can have an impactful contribution in image processing [3].

The information technology industry has been reformed by the entrance of Cloud Computing which allows adaptable and flexible requests for provisioning of computing resources which are supplied over internet on pay per use basis. Many organizations are moving to cloud to cut down their cost of investment on building up large infrastructures because Cloud provide them to dynamically allocate resources without worrying about the maintenance of any kind as cloud providers hosts the underlying infrastructures. There are different types of clouds services such as Infrastructure as a service(IaaS), Platform as a service(PaaS), Software as a service(SaaS), Storage as a service(SEaaS), Security as a service(SECaaS), Data as a service(DaaS), Test environment as a service(TEaaS), Desktop as a service(DaaS), API as a service(APIaaS). Thus Cloud provides *as a service where * denotes any kind of services. Few examples of cloud providers of the three main services are: 


\subsection{IaaS:}

Amazon EC2,Google Compute engine etc delivers computer infrastructure as a utility service, typically in a virtualized environment. That means, it supplies computing resources and storage resources for users.

\subsection{PaaS :}

Google App Engine( GAE),Microsoft Azure etc delivers a platform or solution stack on a cloud infrastructure. It can give users better performance, a more personalized hardware and software services \& a lot of infrastructure module.

\subsection{SaaS :}

Google Apps, IBM blue cloud etc. provides the appliction over the Internet /Intranet by dint of cloud Infrastructure.Using SaaS , users also rent application software and databases.

All these services can be provided by different deployment models, namely- Public Cloud: Sold to the public, mega-scale infrastructure; Private Cloud: enterprise owned or leased; Hybrid Cloud: composition of two or more cloud; and Community Cloud: shared infrastructure for specific community [4].

Grid Computing can be considered a type of distributed computing, where all the resources whether local or global are utilized to provide computational support for a single problem. It is used to provide a high performance computing support.The resources are loosely coupled and which are geographically dispersed unlike cluster computing. Grids are usually dedicated for a single application. But they can also be used for other variety purposes. For this grids are constructed with middleware libraries. Management of resources both local and global becomes a great issue. One such issue is the equal load distribution among all the nodes. Researchers have provided solutions to it from diverse perspective. The concepts of genetic algorithm also provide a probable solution to it.

\section{EVOLUTIONARY ALGORITHM}

Evolutionary algorithm (EA) is a stochastic search based is a subset of EC, a generic based metaheuristics that gives an optimized solution to a problem by improving a candidate solution in an iterative manner. EA employs the mechanism inspired by biological evolution such as reproduction, mutation, recombination and selection.

\subsection{Steps of Evolutionary Algorithm [5][6][7]}

- Given a population of individuals, the environment pressure cause natural selection (Survival of fittest) and grows the fitness of population.

- Each individual is evaluated using a fitness function that is specific to the problem being solved.

- Based upon their fitness value, a number of individual are chosen to be parents.

- New individuals or offspring are produced from those parents by recombination or mutation.

- The fitness value of the offspring and old candidates are competed for a place in the next generation. 
International Journal on Information Theory (IJIT), Vol.3, No.3, July 2014

- This process can be iterated until a solution is found.

\subsection{Gene expressing programming (GEP)}

GEP is an evolutionary algorithm that helps in generating a learning method by creating complex tree structures of computer programs or models. These programs learn to adapt an environment by changing their sizes, shapes, and composition much like a living organism does. The GEP programs are encoded like the living organism in simple linear chromosomes of fixed length. GEP is a genotype that benefits from a simple genome to keep and send out the genetic information it is also a complex phenotype to explore an environment and adapt to it. Thus GEP is a genotype-phenotype system [8].

\subsection{Genetic algorithm (GA)}

Genetic algorithm is a heuristic search that follows the process of natural selection [9][10]. The algorithm is routinely used to get optimized solution and in search problems [11]. The technique of natural evolution like inheritance, mutation, selection and crossover inspires GA.

\subsection{Genetic programming (GP)}

Genetic programming is another evolutionary algorithm based methodology employing the same biological evolution to uncover computer programs performing a user defined task [12]. According to a fitness function determined by a program, a population of computer programs can be optimized and thereby perform a given computational task [13]. GP is computationally very intensive and so mainly used to solve relatively simple problems. It has produced many novel and outstanding results in areas like quantum computing electronic design, game playing, image processing etc.

\subsection{Evolutionary programming (EP)}

EP is related to genetic programming with no fixed structure and the main variation operator is mutation wherein GP there is fixed program structure and only its numerical parameters are evolving [14][15]. In EP each specific species are seen as the members of population and therefore each parent generates an offspring.

\subsection{Evolution strategy (ES)}

ES is also based on the ideas of adaption and evolution. The natural problem-dependent representations are used where mainly mutation and selection act as a search operator. Here too the operators are also applied in a loop. Each iteration of the loop is called a generation. Until a termination criterion is made the sequence of generation is continued [16][17].

\subsection{Differential evolution (DE)}

$\mathrm{DE}$ is a stochastic optimization method which minimizes an objective function that can mold the problem's objectives while incorporating constraints [18]. The three main advantages of DE: finding the true global minimum regardless of the initial parameters values, fast convergence, and using a few control parameters [19].

\subsection{Differential search algorithm (DSA)}

It is used for solving real valued numerical optimization problems. It follows migration of super organism utilizing the concept of stable motion [20]. It is a multi strategy based advanced 
evolutionary algorithm that analogically simulates a super organism migrating between two stop over.

\subsection{Application of Genetic Algorithm}

\subsubsection{Contribution of GA in Cloud Computing in Solving Scheduling Problems:}

Jinhua et. al. [21] proposed genetic algorithm as a scheduling strategy for load balancinsg of virtual machine(VM) resources. In Cloud Computing there are two types of scheduling: the first type is task scheduling where tasks are mapped into VM and the second type is VM scheduling which is also known as resources scheduling. In most of the cases resource scheduling in Cloud considers only the current state of the system but hardly considers system variation and historical data and as a result of which load imbalance of the system occurs. Therefore, to tackle this problem in VM resource scheduling the authors in [21] used genetic algorithm by considering historical data and present state of the system and their strategy helped in computing in advance the influence on the system once the required VM resources were deployed and then picks the least affective solution which resulted in best load balancing and at the same time avoided dynamic VM migration thereby reducing the cost of migration.

Shailesh Sawant [22] proposed a GA based VM resource scheduling strategy that focuses on system load balancing which is similar to the work done in [21] where the GA approach finds in advance the effect of the deployment of new VM resources in the system. The traditional algorithms when used for resource scheduling ends up in imbalance of load and also the number of VM migration also increases. The author carried out an experiment to test the performance of the overall strategy by using most popular open source Virtual machine management infrastructure 'Open Nebula' and outcome of the experiment showed that, this technique can better realize proper load balancing and system resource utilization.

Sandeep Tayal [23] proposed a Fuzzy-GA for optimization of scheduling decision where in a job queue the entire group of task are evaluated by a two levels task scheduling mechanism which fully utilizes the resources and also fulfills the user's requirements.

\subsubsection{Particle swarm optimization(PSO) [28]}

Particle swarm optimization technique was first developed by Dr. Eberhart and Dr. Kennedy in the year 1995. It is a stochastic optimization technique, inspired from flocking birds and fish schooling. PSO imitates human or insects social behavior. A procedure/theory often followed by flocking birds consists of the following:

- Steering towards the centre

- Match with the neighbor's velocity

- Avoid collisions

In PSO, each single solution is a "bird" in the search space. We recognise that as a particle. All of particles have fitness values which are evaluated by the fitness function to be optimized, and have velocities which direct the flying of the particles. The particles fly through the problem space by following the current optimum particles. In order to maintain a balance between exploration and exploitation, a PSO system combines local search methods with global search methods. This can be seen in the case of flocking birds, where every bird tries to maintain its velocity considering both its own velocity and of its neighboring bird. Thus we can say that in a PSO system, particles fly around in a multidimensional search space. During flight, each particle adjusts its position 
International Journal on Information Theory (IJIT), Vol.3, No.3, July 2014

according to its own experience, and according to the experience of a neighboring particle, making use of the best position encountered by itself and its neighbor.

\subsubsection{Honeybee algorithm[29][30]}

As the name goes, this algorithm has been inspired from the food foraging behavior of honey bees. It is a combination of neighborhood search and random search and finds its use in various optimization problems. An initial search for food by honey bees usually involves the process of a random search from one flower patch to another. They tend to evaluate the patches based on the nectar quality, and the energy usage. When a particular bee is capable of finding a source of food, they return to their hive and circulate this information to the other honey bees. This information usually involves three important points. They are, the direction of the food source, the distance to the food source and the quality rating (fitness) of the food. According to the fitness, more no of bees can visit the patches or they can abandon it. This information is helpful for the colony to send the other bees to that particular source. Implementing this concept in searching for an optimal solution in a search space will involve the following steps:

- We first initialize a population of agents (scout bees) for a random search.

- After this we need to specify the size of the neighborhood.

- The agents (bees) are to be recruited at specific sites.

- The fittest bees (fittest/optimal solution) are selected among them.

- The remaining bees are then again assigned to do a random search.

Some applications of this algorithm include statistical quality control, clustering, job scheduling, data mining, Travelling Salesman Problem (TSP) etc.

\subsubsection{Cuckoo Search (CS)[31]}

This concept has been taken and inspired from the brood parasitism of cuckoo birds. Cuckoo birds never build their own nests. They tend to lay their eggs in nests of other birds. Such breeding behaviour of this searching technique and is idealized for various optimization problems. CS uses the following representations:

Every solution is represented by an egg in the nest, and the egg of a cuckoo represents a new solution. The aim of this searching technique is to replace a not-so-good solution in the nests by a comparatively better solution. Its simplest form can be taken as one egg in a nest. The algorithm can be extended to more complicated cases with multiple eggs in multiple nests.

CS has the following rules:

- A nest is chosen at random and each cuckoo lays one egg at a time.

- The best solutions are represented by best nests with good quality eggs in it. These set of solutions are then carried over to the next generation.

- Hosts nests available are fixed.

- Whenever a cuckoo egg is discovered by a host, they are considered as worst solutions. They are dumped and not taken further to the next generation.

- A particular host can discover a cuckoo egg with a probability of $\mathrm{p}(0,1)$.

3.9.5 Contribution of Swarm Intelligence algorithm in Cloud Computing, Grid Computing and Image Processing : 
The problem of task and resource scheduling in both cloud computing and grid computing is an NP-hard optimization problem which can be solved by using some meta-heuristic techniques, therefore swarm intelligence algorithms are widely used for such load balancing to achieve optimal machine utilization. The artificial ant swarm can be used to effectively perform the feature extraction in digital images [32] as well as in image segmentation [3]. It is also helpful in multi-level thresholding using meta-heuristic techniques. There is several swarm intelligence approaches applied in data clustering which are used along with clustering techniques like kmeans algorithm [32]. Some of the applications are discussed as follows:

\subsubsection{Application of ACO and Honeybee Algorithm in Cloud Computing:}

Kun Li et.al.[33] proposed a task scheduling strategy in cloud computing which was based on Load Balancing Ant Colony Optimization (LBACO) algorithm with the objective of optimize the makespan of all the tasks set provided. The authors simulated their approach using the CloudSim toolkit package and the experiment results indicated that the proposed LBACO algorithm surpassed First Come First Serve(FCFS) and the basic ACO (Ant Colony Optimization).

K. Mukherjee et.al. [34] proposed eco-friendly algorithm by combining both honey bee and ant colony algorithm in cloud computing which reduced the operational cost by minimizing power consumption which also diminished global warming to a great extend. The proposed Bee-Ants colony system was used for proper energy efficient resource management where initially the jobs are divided into two parts; the first part which looks after the proper management of overloaded and under loaded Central Processing Unit (CPU) (service rescheduling) was carried out by honey bee algorithm and the second part, which helps to manage the idle CPUs (power consumption management) ant colony algorithm. The authors have validated their study by a set of experiments using Ubuntu's 10.04 Server editions by setting up a small private cloud consisting of two servers.

Dhinesh Babu et. al.[35] proposed an algorithm which was inspired by the honey bee behavior for load balancing (HBB-LB) to maximize the throughput by balancing the load uniformly over all set of virtual machines. This approach demonstrated a significant improvement in average execution time and reduction in waiting time of tasks on queue as it works very efficiently for heterogeneous systems in cloud computing by balancing independent non-preemptive tasks.

\subsubsection{Application of ant-colony optimization in grid load balancing}

In [36] we get to know of a new ant colony approach to grid load balancing or to save the nodes from getting overloaded. Their proposed model is capable of distributing the load equally among all nodes by incorporating a concept of transferring a coloured colony by each node. By this the ants/loads with same nests/characteristics donot get attracted to follow the same path/node, but get distributed evenly to other colonies/nodes as well. However this was implemented taking small number of nodes and short jobs.

In [36] we come to know about yet another approachwhich has some similarity to ants to solve load balancing in dynamic grid environment. Their proposed model consists of two types of agents. The updates regarding the node and the load are performed by these agents either on their way forward or their way back to the server.

\subsubsection{Application of Ant Colony Optimization and Honeybee Algorithm in Image Processing:}

In the work of Zhuang et. al., using ant colony system a perceptual graph is generated representing the relationship between adjacent image points. From this graph the image features 
International Journal on Information Theory (IJIT), Vol.3, No.3, July 2014

are obtained. In one of the clustering processes, ant colony was used to obtain initial cluster centers for k-means algorithm by Sara Ssaatchi et. al. to reduce the segmentation computation producing more accurate and stabilized results.

By specifying intensity edges pixels an automatic image enhancement using honey bee was implemented by Jaspreet Kaur et. al. They also showed that honey bee yielded better results than PSO in terms of maximization of pixel numbers and pick signal to noise ratio [37].

\section{CONCLUSION}

In this paper, various EC techniques have been discussed focusing on their applications in image processing, cloud computing and grid computing. In the field of image processing, using an optimized search space acquired with the help of EC techniques benefits in image compression, image enhancement, feature extraction, and pattern recognition resulting into robust, accurate and stable performance. Similarly for cloud and grid computing, EC techniques are used in solving various kinds of problems such as load balancing, task scheduling, resource scheduling, reduction in energy consumption, etc. Thus in a nutshell EC techniques are extensively and effectively used for getting robust results by optimization.

\section{REFERENCES}

[1] J.E. Smith , Agoston E. Eiben, "Introduction to Evolutionary computation" Alex M. Andrew, Robotica, Vol. 22, 2004

[2] Evolutionary algorithm http://webmining.spd.louisville.edu/Websites/COMB-OPT/.../LectureEC.pdf[Online]

[3] Sara Saatchi and Chih-Cheng Hung,"Swarm Intelligence and Image segmentation", Swarm Intelligence:Focus on Ant and Particle Swarm Optimization, ISBN 978-3-902613-09-7, pp.532, December 2007

[4] Bhaskar Prasad Rimal, Eumni Choi, Ian Lumb, “A Taxonomy and Survey of Cloud Computing System", Fifth International Joint Conference on INC,IMS and IDC, 2009.

[5] T.Back. Evolutionary Algorithms in Theory and Practice. Oxford University Press, New York, 1996.

[6] Th. Back and H-P Schwefel. An overview of Evolutionary Algorithms for parameter optimisation. Evolutionary Computation , 1(1):1-23, 1993.

[7] A.E. Eiben. Evolutionary computing : the most powerful problem solver in the universe? Dutch Mathematical Archive (Nederlands Archief Voor Wiskunde), 5/3(2) : 126-131,2002

[8] GENE EXPRESSING http://en.wikipedia.org/wiki/Gene_expression_programming [Online] 2012.

[9] GENETIC ALGORITHM, http://en.wikipedia.org/wiki/Genetic_algorithm [Online].2014

[10] GENETIC ALGORITHMS http://www.doc.ic.ac.uk/ nd/surprise_96/journal/vol1/hmw/article1.html [Online].2014

[11] David Sinriech and Abekasis Meir, Process selection and tool assignment in automated cellular manufacturing using Genetic Algorithms, Annals of Operations Research 1998, Volume 77, Issue 0, pp 51-78

[12] GENETIC PROGRAMMING, http://en.wikipedia.org/wiki/Genetic_programming [Online] 2014.

[13] Stephanie Forrest, Genetic Algorithms: Principles of Natural Selection Applied to Computation, Science, NewSeries, Vol. 261, No. 5123 (Aug. 13,1993), 872-878.

[14] GENETIC ALGORITHM, http://en.wikipedia.org/wiki/Gene_expression_programming [Online]2014.

[15] Kaisa Miettinen, P. Neittaanmaki, Evolutionary Algorithms in Engineering and Computer Science: Recent Advances in Genetic Algorithms, Evolution Strategies, Evolutionary Programming, GE ,John Wiley \& Sons, Inc. New York, NY, USA@1999, ISBN:0471999024

[16] Hans-Georg Beyer, Hans-Paul Schwefel, Evolution Strategies A Comprehensive Introduction, Natural Computing 1:3-52,2002 @ 2002 Kluwewr Academic Publishers. 
[17] Daan Wierstra, Tom Schaul, Jan Peters, Juergen Schmidhuber, Natural Evolution Strategies, http://www.idsia.ch/ daan/papers/cec08.pdf

[18] A.K. Qin, V.L. Huang, P.N. Suganthan, Differential Evolution Algorithm With Strategy Adaptation for Global Numerical Optimization, IEEE Transactions on Evolutionary Computation, Vol. 13, No.2, April 2009

[19] Karaboga, Dervis, Okdem, Selcuk, A Simple and Global Optimization Algorithm for Engineering Problems: Differrential Evolution Algorithm, Turkish Journal of Electrical Engineering \& Computer Sciences 2004, Vol. 12 Issue 1,p53-60.8p. 1Diagram, 3 Charts, 5 Graphs.

[20] DIFFERENTIAL SEARCH http://www.mathworks.in/matlabcentral/fileexchange/43390-differential-search-algorithm-amodernized-particle-swarm-optimization-algorithm. [Online] 2013.

[21] Jinhua Hu, Jianhua Gu, Guofei Sun, Tianhai Zhao (2010). A Scheduling Strategy on Load Balancing of VirtualMachine Resources in Cloud Computing Environment, IEEE Third International Symposium on Parallel Architectures, Algorithms and Programming (PAAP), pp. 89 - 96.

[22] Shailesh Sawant (2011). A Genetic Algorithm Scheduling Approach for Virtual Machine Resources in a Cloud Computing Environment, Master's Projects. Paper 198. http://scholarworks.sjsu.edu/etd_projects/198

[23] Sandeep Tayal(2011). Tasks Scheduling optimization for the Cloud Computing Systems, International Journal Of Advanced Engineering Sciences And Technologies, Vol 5( 2):111 - 115.

[24] Sankar K.P., and Wang, P.P.,eds, "genetic algorithm for pattern recognisatio“" Boca Raton London: CRC Press.1996.

[25] S. Prakash, D. P. Vidyarthi , "Load Balancing in Computational Grid Using Genetic Algorithm ",Advances in Computing: 2011; 1(1): 8-17 DOI: 10.5923/j.ac.20110101.02

[26] SWARMINTELLIGENCE. http://en.wikipedia.org/wiki/Swarm_intelligence. [Online]. 2014

[27] ANTCOLONY OPTIMIZATION. http://en.wikipedia.org/wiki/Ant_colony_optimization_algorithms. [Online]. 2014

[28] PARTICLE SWARM OPTIMIZATION. http://en.wikipedia.org/wiki/Particle_swarm_optimization. [Online]. 2014.

[29] Pham DT, Ghanbarzadeh A, Koc E, Otri S, Rahim S and Zaidi M. The Bees Algorithm. Technical Note, Manufacturing Engineering Centre, Cardiff University, UK, 2005.

[30] Karaboga.D, (2005). An idea based on honey bee swarm for numerical optimization. Technical Report TR06, Erciyes University, Engineering Faculty, Computer Engineering Department, 2005.

[31] CUCKOO SEARCH. http://en.wikipedia.org/wiki/Cuckoo_search. [Online]. 2014.

[32] X.D Zhuang and N.E Mastorakis, Image Processing with the Artificial Swarm Intelligence, Advances in Image Analysis -Nature Inspired Methodology, WSEAS Press, ISBN:978-960-474-290-5.

[33] Kun Li, Gaochao Xu, Guangyu Zhao, Yushuang Dong, Dan Wang (2011), Cloud Task scheduling based on Load Balancing Ant Colony Optimization, IEEE Sixth Annual China grid Conference (ChinaGrid), pp. 3 - 9

[34] K.Mukherjee, G.Sahoo(2010), “Green Cloud: An Algorithmic Approach”, International Journal of Computer Applications (0975 - 8887), Volume 9- No.9, November 2010.

[35] Dhinesh Babu L.D and P. Venkata Krishna(2013), "Honey bee behavior inspired load balancing of tasks in cloud computing environments", Applied Soft Computing 13 (2013) 2292-2303, Journal of Elsevier, journal home page: www.elsevier.com/locate/asoc

[36] Simone A. Ludwig • Azin Moallem, "Swarm Intelligence Approaches for Distributed Load Balancing on the Grid", Department of Computer Science University of Saskatchewan, Canada.

[37] Jaspreet Kaur, Sukwinder Kaur, and Maninder Kaur, Image Enhancement Using Particle Swarm Optimization and Honey Bee, IJAR Vol.2 Issue 2, ISSN:2278-7844

\section{Biography Of The Authors}

\section{Rajkumari Bidyalakshmi Devi}

She is presently pursuing M.tech (Computer Science \& Engineering) in Assam Don Bosco University, Guwahati, Assam, India. She has acquired B.E (Computer Science) degree in the year 2010 from Manipur Institute Of Technology under Manipur University, Manipur, India. Her major research area is in Image Processing for Object Detection. She has presented paper in National Conferences.

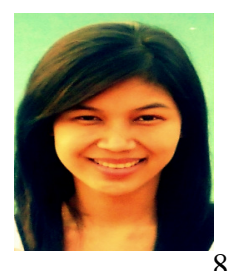


Esha Barlaskar

She is presently pursuing M.Tech (Computer Science \& Engineering) in Assam Don Bosco University, Guwahati, Assam, India. She has acquired B.E. (Computer Science) degree in the year 2010 from North-Eastern Hill University, Meghalaya, India. Her major research areas are Cloud Computing and Artificial Intelligence. She has published papers in National and International Conferences as well as journals.

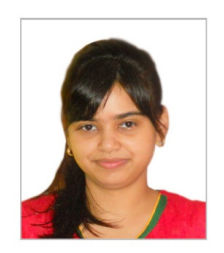

Oinam Binarani Devi

She is presently persuing M.tech (Computer Science \& Engineering) in Assam Don Bosco University, Guwahati, Assam, India. She has acquired B.E (Computer Science) degree in the year 2012 from PGP College of Engineering \& Technology under Anna University, Chennai, India. Her major research area is Video based Image Processing. She has presented paper in National Conferences.

\section{Smriti Priya Medhi}

She is presently pursuing M.Tech.(Computer Science Engineering) from Assam Don Bosco University, Guwahati, Assam, India. She has acquired B.Tech. (Computer Science) degree in the year 2012 from Assam Don Bosco University. Her major research areas are High Performance Computing Approaches and Artificial Intelligence.

\section{Reingayung Ronra Shimray}

$\mathrm{He}$ is presently pursuing M.tech (Computer Science \& Engineering) in Assam Don Bosco University, Guwahati, Assam, India. She has acquired B.E (Computer Science) degree in the year 2011 from Manipur Institute Of Technology under Manipur University, Manipur, India. His major research area is in Image Processing for character recognition.
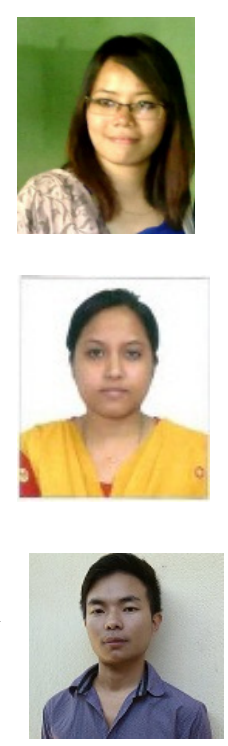